\title{
Magnetostructural coupling, magnetic ordering, and cobalt spin reorientation in metallic $\mathrm{Pr}_{0.5} \mathrm{Sr}_{0.5} \mathrm{CoO}_{3}$ cobaltite
}

\author{
José Luis García-Muñoz, ${ }^{1, *}$ Jessica Padilla-Pantoja, ${ }^{1}$ Xavier Torrelles, ${ }^{1}$ Javier Blasco, ${ }^{2}$ Javier Herrero-Martín, ${ }^{3}$ Bernat Bozzo, ${ }^{1}$ \\ and José A. Rodríguez-Velamazán ${ }^{2,4}$ \\ ${ }^{1}$ Institut de Ciència de Materials de Barcelona (ICMAB-CSIC), Campus universitari de Bellaterra, \\ E-08193 Bellaterra, Spain \\ ${ }^{2}$ Instituto de Ciencia de Materiales de Aragón, CSIC-Universidad de Zaragoza, 50009 Zaragoza, Spain \\ ${ }^{3}$ ALBA Synchrotron Light Facility, 08290 Cerdanyola del Vallès, Barcelona, Spain \\ ${ }^{4}$ Institut Laue-Langevin (ILL), BP 156, 38042 Grenoble Cedex 9, France
}

(Received 19 March 2016; published 11 July 2016)

\begin{abstract}
In half-doped $\operatorname{Pr}_{0.50} A_{0.50} \mathrm{CoO}_{3}$ metallic perovskites, the spin-lattice coupling brings about distinct magnetostructural transitions for $A=\mathrm{Ca}$ and $A=\mathrm{Sr}$ at temperatures close to $\sim 100 \mathrm{~K}$. However, the ground magnetic properties of $\operatorname{Pr}_{0.50} \mathrm{Sr}_{0.50} \mathrm{CoO}_{3}$ (PSCO) strongly differ from $\operatorname{Pr}_{0.50} \mathrm{Ca}_{0.50} \mathrm{CoO}_{3}$ ones, where a partial $\operatorname{Pr}^{3+}$ to $\operatorname{Pr}^{4+}$ valence shift and $\mathrm{Co}$ spin transition makes the system insulating below the transition. This paper investigates and describes the relationship between the Imma $\rightarrow I 4 / \mathrm{mcm}$ symmetry change [Padilla-Pantoja, García-Muñoz, Bozzo, Jirák, and Herrero-Martín, Inorg. Chem. 53, 12297 (2014)] and the original magnetic behavior of PSCO versus temperature and external magnetic fields. The FM1 and FM2 ferromagnetic phases, above and below the magnetostructural transition $\left(T_{S 1} \sim 120 \mathrm{~K}\right)$ have been investigated. The FM2 phase of PSCO is composed of [100] FM domains, with magnetic symmetry $I m^{\prime} m^{\prime} a\left(m_{x} \neq 0, m_{z}=0\right)$. The magnetic space group of the FM1 phase is $F m^{\prime} m^{\prime} m$ (with $m_{x}=m_{y}$ ). Neutron data analyses in combination with magnetometry and earlier reports results agrees with a reorientation of the magnetization axis by $45^{\circ}$ within the $a b$ plane across the transition, in which the system retains its metallic character. The presence below $T_{S 1}$ of conjugated magnetic domains, both of $F m^{\prime} m^{\prime} m$ symmetry but having perpendicular spin orientations along the diagonals in the $x y$ plane of the tetragonal unit cell, is at the origin of the anomalies observed in the macroscopic magnetization. A relatively small field $\mu_{0} H[\perp z] \gtrsim 30 \mathrm{mT}$ is able to reorient the magnetization within the $a b$ plane, whereas a higher field $\left(\mu_{0} H[\| z] \gtrsim 1.2 \mathrm{~T}\right.$ at $\left.2 \mathrm{~K}\right)$ is necessary to align the Co moments perpendicular to the $a b$ plane. Such a spin reorientation, in which the orbital and spin components of the Co moment rotate joined by $45^{\circ}$, was not observed previously in analogous cobaltites without praseodymium.
\end{abstract}

DOI: 10.1103/PhysRevB.94.014411

\section{INTRODUCTION}

Following the extensive investigations on the nature of the spin-state (SS) changes in undoped $L n \mathrm{CoO}_{3}$ compounds, the SS of trivalent cobalt is being examined in a variety of cobaltites because of its proved ability to condition the transport, magnetic, and electronic properties of compounds like the $\left(L n_{1-y} L n_{y}^{\prime}\right)_{1-x} A_{x} \mathrm{CoO}_{3}\left(L n, L n^{\prime}\right.$ : lanthanides, $A$ : alkaline-earth) perovskites [1-4]. In this context, the physical properties of half-doped Pr-based $\operatorname{Pr}_{0.50} A_{0.50} \mathrm{CoO}_{3}$ specimens are attracting the interest due to the observation of nonconventional phase transitions and distinct unexpected properties with $A=\mathrm{Ca}$ [4-11] and $\mathrm{Sr}$ [12-18].

In this way, $\mathrm{Ca}$ doped $\mathrm{Pr}_{0.50} \mathrm{Ca}_{0.50} \mathrm{CoO}_{3}$ [and other related $(\mathrm{Pr}, L n)_{1-x} \mathrm{Ca}_{x} \mathrm{CoO}_{3}$ cobaltites near half-doping $(x \sim 1 / 2)$ ] exhibits an exotic metal-insulator transition [1] produced by two concurrent phenomena: (i) an abrupt $\mathrm{Co}^{3+} \mathrm{SS}$ change [10] and (ii) a partial $\mathrm{Pr}^{3+}$ to $\mathrm{Pr}^{4+}$ valence shift [7-9]. $\mathrm{Pr}_{0.50} \mathrm{Ca}_{0.50} \mathrm{CoO}_{3}$ (PCCO) is orthorhombic (Pnma) and metallic, but it becomes insulating at $T_{\mathrm{MI}} \sim 80 \mathrm{~K}$. An electron is transferred from some Pr atoms to Co sites [6-10], and a concomitant SS crossover promotes the stabilization of the $\mathrm{Co}^{3+}$ low spin (LS) state [10]. Remarkably, PCCO exhibits exceptional

\footnotetext{
*Corresponding author: garcia.munoz@icmab.es
}

photoresponse capabilities of potential interest for ultrafast optical switching devices [11]. The generation of metallic domains in the sample after photoirradiation in the nonconducting state occurs thanks to the strong connection between volume expansion, electron mobility, and excited SSs [10].

The structural, magnetic, and electronic properties of $\mathrm{Pr}_{0.50} \mathrm{Sr}_{0.50} \mathrm{CoO}_{3}$ (PSCO) apparently differ from PCCO (without magnetic order due to the LS state stabilization in the trivalent Co sites). PSCO is ferromagnetic (FM) below $T_{\mathrm{C}} \sim 230 \mathrm{~K}$ and metallic in all of the temperature range. Mahendiran and Schiffer [12] initially reported unexpected magnetic anomalies at $T_{S 1} \sim 120 \mathrm{~K}$. The discovery of a second magnetic transition and intriguing steplike behavior of the magnetization, which decreases or increases depending on the magnitude of the applied field [12-16], was followed by the detection of structural anomalies at the same temperature by Troyanchuk et al. [15]. The lack of consensus on the structural properties of PSCO led to different structural descriptions, used to justify visible changes in diffraction data at low temperatures [13,15-17]. On the other hand, some works attributed the magnetostructural transition to a phase separation at $T<120 \mathrm{~K}$, proposing a two-phase magnetic state at low temperature [16]. Finally, a reliable description of the crystal structure evolution across $T_{S 1}$ was reported in 2014 by Padilla-Pantoja et al. in Ref. [18]. From the high temperature cubic phase upon decreasing temperature, PSCO follows the Pm-3m $\rightarrow R-3 c \rightarrow I m m a \rightarrow I 4 / m c m$ 
transformations. Hence the symmetry change at the magnetostructural transition at about $T_{S 1}$ implies an orthorhombictetragonal conversion $(\mathrm{O}-\mathrm{T})[18]$.

The absence of this transition in other half-doped cobaltites without $\mathrm{Pr}$ ions and the spontaneous Pr valence shift reported in PCCO and other ( $\mathrm{Pr}, L n)_{1-x} \mathrm{Ca}_{x} \mathrm{CoO}_{3}$ cobaltites motivated to investigate the possible importance of the $\operatorname{Pr} 4 f-\mathrm{O} 2 p$ hybridization for the structural changes in PSCO $[13,16,18]$. Unlike PCCO, a $\mathrm{Pr}^{3+}$ to $\mathrm{Pr}^{4+}$ oxidation process was ruled out in PSCO by means of X-ray absorption spectroscopy (XAS) studies at $\operatorname{Pr} M_{4,5}$ and $\operatorname{Pr} L_{3}$ edges and charge-transfer multiplet calculations [19]. Similarly, XAS measurements of the temperature evolution of the Co $L_{2,3}$ edges showed that the $\mathrm{SS}$ of Co ions remains nearly unaltered across the anomalous transition [19].

Evidences of the interplay between the magnetic and crystal structures were obtained from transverse susceptibility and magnetostriction measurements, which point to likely changes in the magnetocrystalline anisotropy at $T_{S 1}$ [12-14,20]. Lorentz transmission electron microscopy (LTEM) images reported by Uchida et al. also suggested a reorientation of the magnetization axis by $45^{\circ}$ when studying the evolution of the magnetic domain structure under electron beam [21]. The importance of the spin-lattice coupling has been also confirmed by means of x-ray magnetic circular dichroism (XMCD) experiments at the Co $L_{2,3}$ edges [22]. They reveal a sizeable orbital momentum in Co atoms that evolves in like manner as the atomic spin moment (or the macroscopic magnetization) across the two successive magnetic transitions, pointing to a coupling between the ordered electronic spins and the orbital states of $3 d$ electrons.

We present a neutron diffraction investigation of the singular magnetic properties of $\mathrm{Pr}_{0.50} \mathrm{Sr}_{0.50} \mathrm{CoO}_{3}$ that clarifies the temperature and field evolution of the magnetic symmetry in this system. The relevance of the structural symmetry changes on its magnetic behavior has been elucidated.

\section{EXPERIMENTAL DETAILS}

Polycrystalline ceramic samples of PSCO were prepared by the conventional solid-state reaction method under an oxygen atmosphere, as reported in Ref. [18]. High-purity $\mathrm{Co}_{3} \mathrm{O}_{4}$ and $\operatorname{Pr}_{6} \mathrm{O}_{11}$ oxides and $\mathrm{SrCO}_{3}$ were used as precursors. The last two annealings were performed at $1100^{\circ} \mathrm{C}$ (for $12 \mathrm{~h}$ ) and $1170^{\circ} \mathrm{C}$ (for $24 \mathrm{~h}$ ) under $\mathrm{O}_{2}$, making a slow cooling. Powder samples and compacted pellets were used for the measurements. Samples quality was checked by x-ray diffraction patterns collected at room temperature using a Siemens D-5000 diffractometer and $\mathrm{Cu} \mathrm{K} \alpha$ radiation. They were single phase and free from impurities. The magnetic response to dc and ac magnetic fields was measured using a Superconducting Quantum Interferometer Device (SQUID) and Physical Properties Measuring System (PPMS) from Quantum Design. The latter was also used for electrical transport measurements using the four-probe method and silver paste.

Neutron diffraction experiments were carried at the highflux reactor of the Institut Laue-Langevin (Grenoble) using the $\mathrm{D} 20(\lambda=1.87 \AA)$ and $\mathrm{D} 1 \mathrm{~B}(\lambda=2.52 \AA)$ instruments. Neutron powder diffraction (NPD) measurements in the D20 diffractometer of the ILL were performed using a high take-off angle of $118^{\circ}$ for the $\mathrm{Ge}(115)$ monochromator and a radial oscillating collimator that precedes a microstrip PSD detector, covering an angular range of $150^{\circ}$. In this range, high resolution data as a function of temperature was obtained warming the sample in a cryofurnace from $15 \mathrm{~K}$ up to $443 \mathrm{~K}$. In ramp mode, the temperature shift for individual scans was smaller than $5 \mathrm{~K}$. Additional NPD patterns were also recorded at fixed selected temperatures. A cryomagnet was used on D1B to apply magnetic fields up to $5 \mathrm{~T}$. All the structural and magnetic Rietveld refinements were made using the FullProf program [23].

Crystallographic tools from the Bilbao Crystallographic server were also used [24-26]. A detailed structural study of a PSCO powder sample is reported in Ref. [18]. Temperature dependent XMCD measurements at the Co $L_{2,3}$ edges were also performed on the samples at BL29-BOREAS beamline in the ALBA Synchrotron Light Facility, and the results can be found in Ref. [22].

\section{RESULTS AND DISCUSSION}

The ac susceptibility was measured under a dc field of 75 Oe, superimposed to an ac field of 10 Oe. As illustrated in Fig. 1(a), the magnetic transitions produce on cooling pronounced upturns in the real component of the ac susceptibility $\left(\chi^{\prime}\right)$, forming two separated peaks with maxima at 92 and $225 \mathrm{~K}$, respectively. Regarding the onset of the two peaks in $\chi^{\prime}(T)$, the first one starts to develop at $\sim 245 \mathrm{~K}$ and the second at $\sim 120 \mathrm{~K}$. Magnetization $(M)$ was also measured as a function of temperature and applied magnetic field using a commercial SQUID. A comparison of the zero-field-cooled (ZFC) and field-cooled (FC) magnetizations under $1 \mathrm{kOe}$ plotted in Fig. 1(b) shows a similar hump in both $M(T)$ curves at about $T_{S 1} \sim 120 \mathrm{~K}$. A small splitting in the curves is detected below $T_{\mathrm{C}} \approx 230 \mathrm{~K}$, which increases below $T_{S 1}$. Moreover, it was reported in earlier works that the sharp decrease in $M(T)$ when cooling under small fields is accompanied by thermal hysteresis $[12,13]$.

The metallic resistivity is depicted in the inset of Fig. 1(b). The temperature evolution of the coercive field $\left(H_{c}\right)$ was determined from the hysteresis loops recorded at diverse temperatures. The obtained $H_{c}(T)$ curve is represented in Fig. 1(c). Overall, $H_{c}$ increases with decreasing the temperature, but a clear anomaly (peak shape) is observed at $T_{S 1}$. In the inset, the field dependence of the magnetization is plotted at two temperatures representative of the two FM phases. From now on, we will name FM1 to the distinctive FM state of the $I 4 / \mathrm{mcm}$ phase $\left(T<T_{S 1}\right)$ and FM2 to the FM state of the Imma cell below the Curie temperature $\left(T_{S 1}<T<T_{\mathrm{C}}\right)$. The maximum value of the magnetization $M(H)$ below $T_{S 1}$ (at $5 \mathrm{~K}$ and $7 \mathrm{~T}$ applied field) was very close to $2 \mu_{\mathrm{B}} / \mathrm{f}$.u. ( $M=1.96 \mu_{\mathrm{B}} /$ f.u.) [22].

The temperature dependence of the magnetization measured on cooling under field is shown in Fig. 2(a) for seven distinct magnetic fields. The anomalous steplike behavior of the magnetization around $T_{S 1} \sim 120 \mathrm{~K}$ shows noticeable field dependence. So, the amplitude and sign (positive or negative) of the abrupt magnetization jumps $(\Delta M)$ exhibit an ostensive dependence on the strength of the external magnetic field. By inspection of the magnetization jumps shown in Fig. 2(a) 

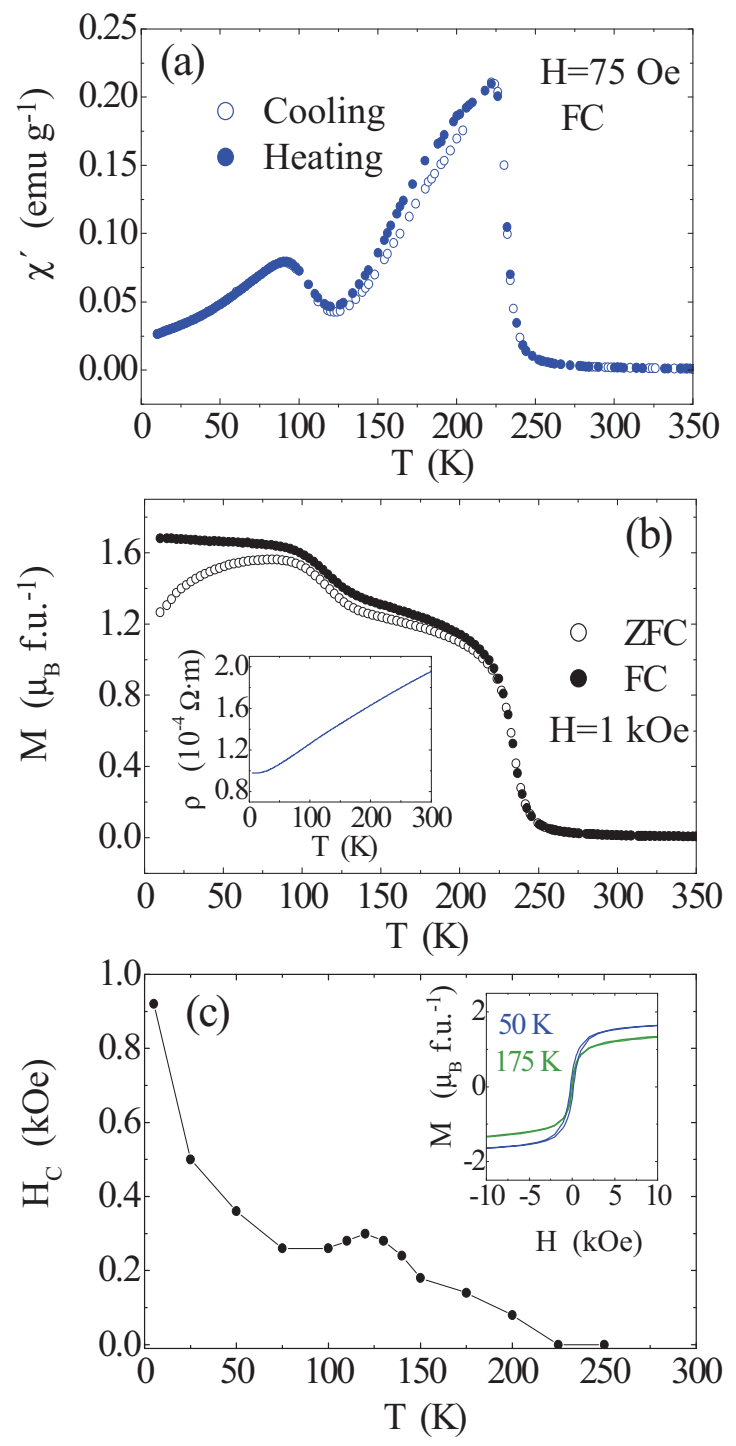

FIG. 1. (a) Real component of ac susceptibility ( $h=10 \mathrm{Oe}$, $13 \mathrm{~Hz}$ ) measured under cooling and heating and a dc field of $75 \mathrm{Oe}$ (FC). (b) FC and ZFC dc magnetization curves (1 kOe). The inset shows the resistivity curve. (c) Temperature evolution of the coercive field $\left(H_{C}\right)$. Inset: field dependence of the magnetization (ZFC) at $175 \mathrm{~K}$ (FM2) and $50 \mathrm{~K}$ (FM1).

for different fields, the corresponding field dependence has been represented in Fig. 2(b), demonstrating a crossover from negative to positive $\Delta M$ values at $T_{S 1}$ at the critical value of $\sim 300 \mathrm{Oe}\left(\mu_{0} H_{\mathrm{cr}} \approx 30 \mathrm{mT}\right)$. The jumps are negative for external fields $H<H_{\text {cr }}$ and positive for $H>H_{\text {cr }}$.

\section{A. FM symmetry in the Imma phase (FM2)}

Below $T_{\mathrm{C}} \approx 230 \mathrm{~K}$, the emergence of FM order produces changes in the neutron profile (FM Imma phase: FM2). Given the smallness of the orthorhombic distortion, it is not easy to unambiguously distinguish the exact orientation of the FM Co moments. However, in this case, the determination of the moments direction is possible, as we will show in this section.

As previously mentioned, a detailed description of the crystal structures of PSCO can be found in Ref. [18].
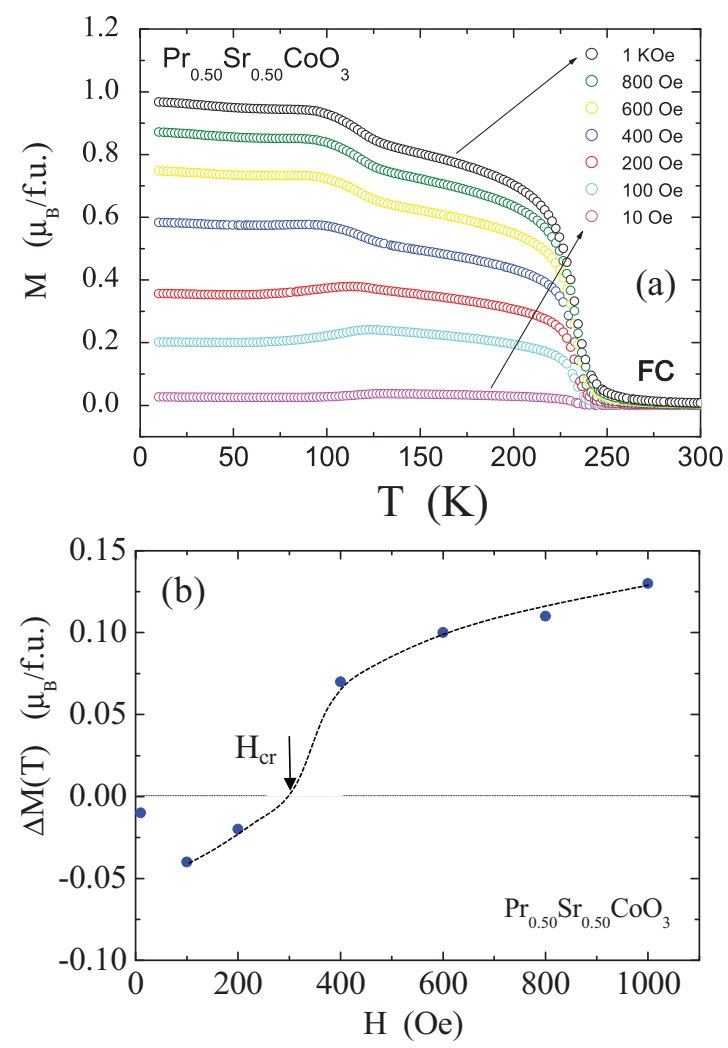

FIG. 2. (a) Temperature dependence of the magnetization measured on cooling at 10, 100, 200, 400, 600, 800, and 1000 Oe (FC). The sign of the magnetization jumps at the second transition are field dependent. (b) Field evolution of the amplitude and sign of the magnetization jumps at the $\mathrm{O}-\mathrm{T}$ transition.

Let us recall first- to avoid possible confusion-that the standard settings of the space groups (SGs) Imma and $I 4 / \mathrm{mcm}$ correspond to different orientations of the perovskite cell: $\sqrt{2} a_{0} \times 2 a_{0} \times \sqrt{2} a_{0}$ in the orthorhombic SG and $\sqrt{2} a_{0} \times \sqrt{2} a_{0} \times 2 a_{0}$ in the tetragonal one. Namely, the longest cell parameter is $b$ in the first setting and $c$ in the second one. To avoid misunderstanding, hereafter we label the FM models $F_{x, y \text { or } z}$, always referring to the unit cell setting of the tetragonal phase, where $z$ denotes the coordinate along the longest axis $2 a_{0}$. Therefore, under this definition, $F_{z}$ in this paper always means that spins are pointing parallel to the longest (vertical) $2 a_{0}$ axis, which is the $c$ axis in $I 4 / \mathrm{mcm}$ but it is the $b$ one in Imma phase.

In an effort to obtain some insight on the anisotropy of the magnetization in the orthorhombic phase, we performed careful refinements of the D20 neutron diffraction pattern at $140 \mathrm{~K}$ using different moment orientations. The differences using different magnetic anisotropies were small. Nevertheless, the best fit was obtained for the $F_{x}$ configuration. $F_{y}$ or $F_{z}$ models generate wrong FM intensities (in comparison to the experimental ones) in well resolved peaks like (123) and (321), being experimentally higher the magnetic contribution to the last. Moreover, these two models also produce an excess of (103) magnetic intensity, whereas the $F_{x}$ configuration nicely reproduces all magnetic intensities.

In addition, several possible magnetic SGs (MSGs) or Shubnikov SGs compatible with the Imma symmetry and 
TABLE I. Magnetic groups and refined magnetic moments in the FM1 (15 K) and FM2 (140 K) phases. See explanation in the text.

\begin{tabular}{lc}
\hline \hline $\begin{array}{l}\text { Magnetic } \\
\text { Space Group }\end{array}$ & $F m^{\prime} m^{\prime} m(\# 69.524)$ \\
\hline Transformation to standard setting & $(-c, a-b,-a-b ; 0,1 / 2,0)$ \\
& Expressed in parent tet. setting: \\
Coordinates & $\left(0,0,0 \mid m_{x}, m_{y}, 0\right)$ \\
for Co moment & $\left(0,0,1 / 2 \mid m_{y}, m_{x}, 0\right)$ \\
& $\left(1 / 2,1 / 2,1 / 2 \mid m_{x}, m_{y}, 0\right)$ \\
& $\left(1 / 2,1 / 2,0 \mid m_{y}, m_{x}, 0\right)$
\end{tabular}

Refined moments (expressed in tet. setting)

Magnetic structure

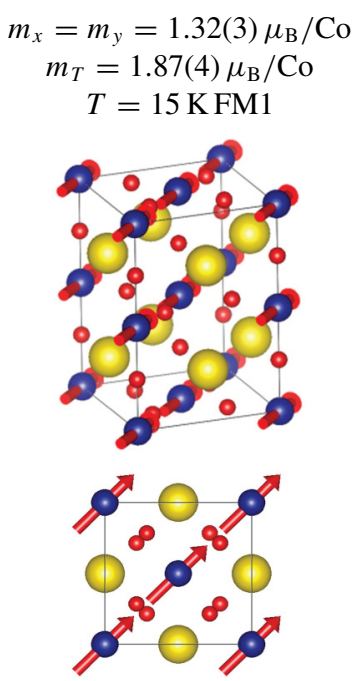

$I m^{\prime} m^{\prime} a(\# 74.558)$

$(a, b, c ; 0,0,0)$

Expressed in parent ort. setting:

$\left(0,0,1 / 2 \mid 0, m_{y}, m_{z}\right)$

$\left(0,1 / 2,1 / 2 \mid 0,-m_{y}, m_{z}\right)$

$\left(1 / 2,1 / 2,0 \mid 0, m_{y}, m_{z}\right)$

$\left(1 / 2,0,0 \mid 0,-m_{y}, m_{z}\right)$

Expressed in tet. setting:

$\left(0,0,0 \mid m_{x}, 0, m_{z}\right)$

$\left(0,0,1 / 2 \mid m_{x}, 0,-m_{z}\right)$

$\left(1 / 2,1 / 2,1 / 2 \mid m_{x}, 0, m_{z}\right)$

$\left(1 / 2,1 / 2,0 \mid m_{x}, 0,-m_{z}\right)$

$$
\begin{gathered}
m_{x}=1.49(3) \mu_{\mathrm{B}} / \mathrm{Co} \\
m_{z}=0
\end{gathered}
$$

$T=140 \mathrm{~K}$ FM2
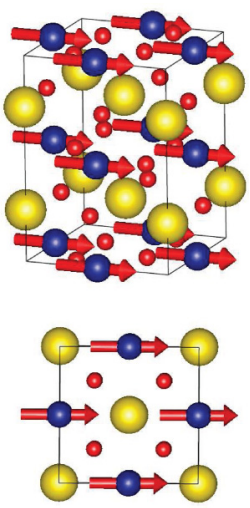

$\mathbf{k}=0$ were considered. Among them, the magnetic subgroups $I m^{\prime} m^{\prime} a$ (allowing $F_{x} G_{z}$, expressed in the tetragonal setting) or $I m m^{\prime} a^{\prime}$ (compatible with $F_{y}$ and $G_{x} F_{z}$, referred to the tetragonal setting) [26,27]. The best fit was obtained with the MSG $I m^{\prime} m^{\prime} a$ [\#74.558, transformation to standard setting: $(\mathbf{a}, \mathbf{b}, \mathbf{c} ; 0,0,0)][25,27]$, compatible with a collinear $F_{x}$ model (tetragonal setting), which yields goodness factors $R_{B}=3.38, R_{f}=2.74, R_{\mathrm{Mag}}=4.89, \chi^{2}=1.58$ (see details in Table I). The Rietveld refinement at $140 \mathrm{~K}$ (above but very close to the O-T phase transition) using the $I m^{\prime} m^{\prime} a$ MSG symmetry converges to the $F_{x}$ model (and $m_{z}=0$, tetragonal setting), and it is plotted in Fig. 3, yielding a FM ordered moment $m_{x}=1.49(3) \mu_{\mathrm{B}} /$ Co. Schematic projections of the magnetic order in the orthorhombic cell are shown in Table I and Fig. 3. The cell parameters and atomic coordinates coincide with those reported in Ref. [18]. So, $a=5.3771(8) \AA$, $b=7.5950(1) \AA, c=5.4320(7) \AA ; \operatorname{Pr} / \mathrm{Sr}(4 e): z[\mathrm{Pr} / \mathrm{Sr}]=$ $-0.0005(4)$; $\mathrm{Co}(4 b) ; \mathrm{O} 1(4 e): z[\mathrm{O} 1]=0.4544(6) ; \mathrm{O} 2(8 g)$ : $y[\mathrm{O} 2]=0.0243(2)$.

Likewise, we confirmed that lower magnetic symmetries allowing FM order out of the main crystallographic axes (either in the $x y$ plane or out of this plane) produce worse results and generate wrong magnetic intensities in reflections like (101), (020), (103), (301), or (123). For example, the subgroup (of $I m^{\prime} m^{\prime} a$ ) $C 2^{\prime} / m^{\prime}$ allows the FM spins to collectively rotate within the $x y$ plane $\left(F_{x} F_{y}\right.$ model, expressed in the tetragonal setting). Using this MSG (forcing identical moments in the split Co orbits), the refinements converge to moments aligned along $x$, with a negligible $y$ component (tetragonal setting). Summarizing, we have been able to discern the $x$ orientation of the magnetic moments in the FM2 phase (MSG $I m^{\prime} m^{\prime} a$, $\left.m_{x} \neq 0\right)$ of the orthorhombic Imma structure. Paying attention to fine details in the magnetic Rietveld refinement when comparing different models, we conclude that only the $F_{x}$ model (tetragonal setting) correctly matches the experimental intensities.

\section{B. Magnetic symmetry and cobalt spin reorientation in the $14 / \mathrm{mcm}$ phase (FM1)}

Figure 4 (left panel) plots the evolution of two main sets of magnetic reflections: $(110) /(002)$ and $(112) /(020)$ at $2 \theta$ near $28^{\circ}$ and $40^{\circ}$, respectively (D20 data, $\lambda=1.87 \AA$ ). The two transitions can be easily identified. The intensities shown in the figure were recorded in absence of an external magnetic field, and their evolution shows a clear decrease at the magnetostructural transition which is similar to the steplike behavior of $M(T)$ measured at low fields. However, the changes observed in the neutron patterns around $120 \mathrm{~K}$ may arise from potentially concurrent structural and magnetic 
intensity changes. Thereby, we show later that the loss of intensity in the left panel curves of Fig. 4 is not due to a decrease of the FM Co moment (as changes in magnetization could suggest), but it simply has a structural origin. Therefore, Fig. 4 is just an illustrative example that the concurrent structural and magnetic changes in the new tetragonal cell favor cross-correlation between these two types of parameters. For that reason, we decided to first explore possible magnetic orders compatible with the new tetragonal symmetry and confront them with neutron data. Neutron patterns in the low-temperature phase do not show new magnetic reflections symptomatic of an antiferromagnetic (AFM) multiple cell (k is 0 ). Nevertheless, the possibility of canted Co moments with possible coexisting FM and AFM coupled components in the tetragonal cell phase was considered too. Among the most probable magnetic symmetries compatible with the $I 4 / \mathrm{mcm}$ cell in absence of an external field, one can include the maximal subgroups: $I 4 / m c^{\prime} m^{\prime}$ (\#140.547), $I b a^{\prime} m^{\prime}$ or $I b^{\prime} a m^{\prime}$ (\#72.544), and $F m^{\prime} m^{\prime} m$ (\#69.524) [27], all of them compatible with a net FM behavior (collinear or noncollinear) from cobalt atoms sharing one single orbit [24-26]. Some other subgroups of the gray magnetic group $G 1^{\prime}(G=I 4 / \mathrm{mcm})$ were ignored because they are not compatible with ordered moments at Co sites (like $I b a^{\prime} m$ or $I b^{\prime} a m$ ) or imply pure antiferromagnetism [like $I 4 / m c m(\# 140.541)]$.

\section{Discerning between out-of-plane and in-plane ordering}

The tetragonal magnetic symmetry $I 4 / m c^{\prime} m^{\prime}$ (allowing only $m_{z}$ components, $F_{z}$ model) was immediately discarded as it clearly generates wrong magnetic intensities. It is shown in Fig. 4 (right panel) that considering Co moments aligned along [001], the experimental magnetic intensities from the (001) $1=2 n$ planes cannot be reproduced. Hence, unlike the FM I4/mcm phase of the manganite $\operatorname{Pr}_{0.50} \mathrm{Sr}_{0.50} \mathrm{MnO}_{3}$

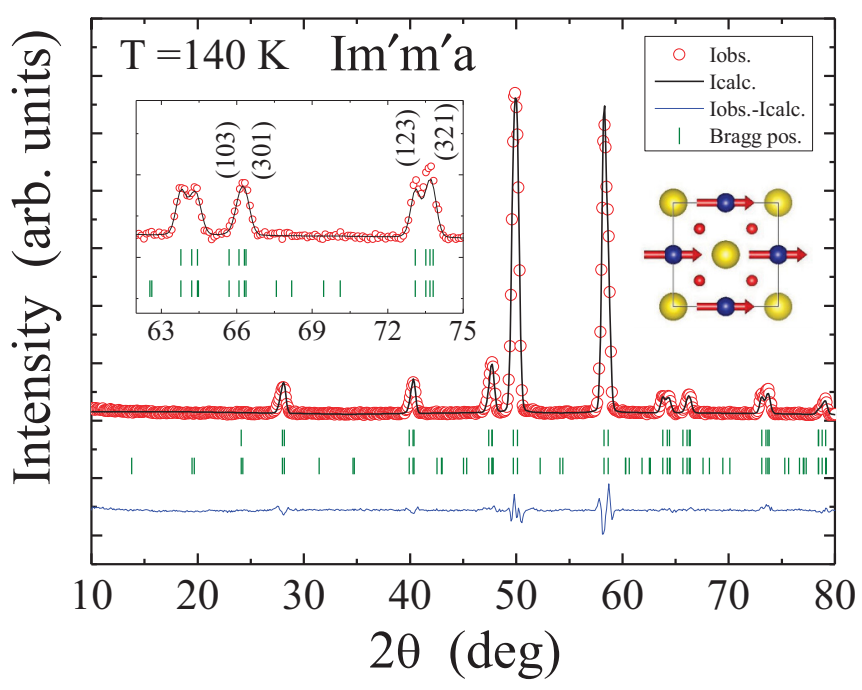

FIG. 3. Rietveld refinement (solid line) of neutron pattern collected at $140 \mathrm{~K}$ (FM2 phase, D20) for the ferromagnetic orthorhombic phase $I^{\prime} m^{\prime} a$. The inset shows indexed magnetic peaks, $\sqrt{2} a_{0} \times a_{0} \times \sqrt{2} a_{0}$ setting. A schematic view of the magnetic structure is also shown (yellow balls are $\mathrm{Pr} / \mathrm{Sr}$ atoms, blue are $\mathrm{Co}$ atoms, and $\mathrm{O}$ atoms are red).
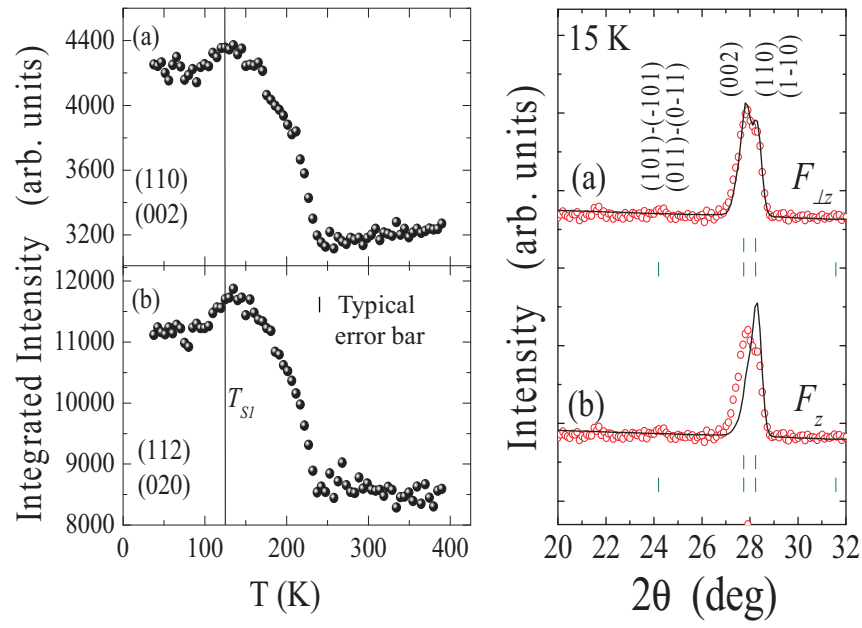

FIG. 4. (Left panel) Evolution of the integrated intensity of two main sets of diffracted reflections with ferromagnetic contribution: (a) (110)/(002) and (b) (112)/(200). Neutron diffraction measurement heating the sample without external field. (Right panel) Selected region of the refined neutron pattern at $15 \mathrm{~K}$ using different orientations for the Co magnetic moments: (a) FM model with in-plane magnetic moments $\left(F_{\perp z}\right.$ model) and (b) FM model with out-of-plane magnetic moments $\left(F_{z}\right.$ model). The reflections are indexed in the $I 4 / \mathrm{mcm}$ setting $\left(\sqrt{2} a_{0} \times \sqrt{2} a_{0} \times 2 a_{0}\right.$ cell $)$.

where the magnetization vector lies along [001] [28], the magnetic moments in the cobalt counterpart compound$\mathrm{Pr}_{0.50} \mathrm{Sr}_{0.50} \mathrm{CoO}_{3}$-are within the $x y$ plane of the tetragonal cell $\left(F_{\perp z}\right)$.

\section{Making compatible neutron and LTEM results}

As the best solution for the magnetic order in the tetragonal phase, we found the $F m^{\prime} m^{\prime} m$ (\#69.524) magnetic SG [transformation to standard setting: $(-\mathbf{c}, \mathbf{a}-\mathbf{b},-\mathbf{a}-\mathbf{b} ; 0,1 / 2,0)]$ with Co having $m_{x}=m_{y}=1.32(3) \mu_{\mathrm{B}} /$ atom as refined values, resulting in Co moments pointing along the diagonal [110] direction within the $a b$ plane of the cell (see Table I). The refinement of the $15 \mathrm{~K}$ pattern with this symmetry is perfectly satisfactory, yielding a FM ordered moment of 1.87(4) $\mu_{\mathrm{B}} / \mathrm{Co}$. Any other tested model did not show better reliability factors: $R_{B}=4.81, R_{f}=3.16, R_{\mathrm{Mag}}=8.55, \chi^{2}=1.59$. Of course, as a matter of fact, the rotation of this magnetic structure around the tetragonal axis does not modify the quality of the neutron refinement. $I b a^{\prime} m^{\prime}$ and $I b^{\prime} a m^{\prime}$ magnetic symmetries correspond to the conjugated magnetic domains associated, respectively, to $F_{x} G_{y}$ and $G_{x} F_{y}$ models (\#72.544), permitting FM order along $x$ or $y$ directions (equivalent in tetragonal symmetry). Nevertheless, the magnetic models with the magnetization vector parallel to the $x$ or $y$ axis of the tetragonal cell $\left(\sqrt{2} a_{0}\right)$ can be discarded because they are not compatible with the low temperature observations made on PSCO by LTEM, reported in Ref. [21], which imply a rotation of the moments by $45^{\circ}$ across the transition. We shall return to this point later. The measured, calculated, and difference profiles for tetragonal PSCO are plotted in Fig. 5. A close view of some refined important magnetic reflections is presented in the inset.

As shown in Table I, the $F m^{\prime} m^{\prime} m$ magnetic symmetry permits two independent components ( $m_{x}$ and $m_{y}$, expressed in 


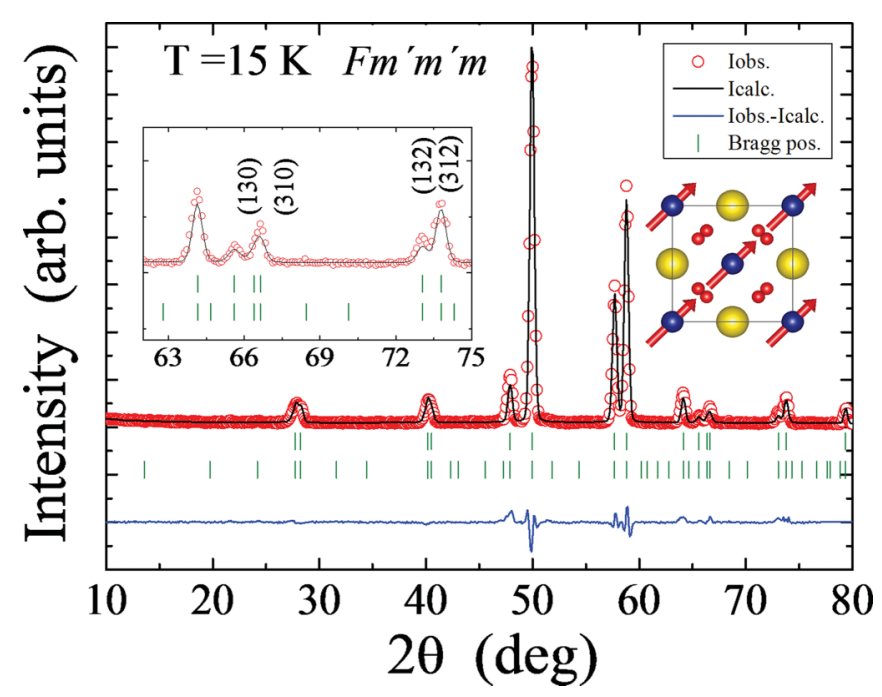

FIG. 5. Rietveld refinement (solid line) of the neutron pattern collected at $15 \mathrm{~K}$ (FM1 phase, D20) for the $14 / \mathrm{mcm}$ structure using the $F m^{\prime} m^{\prime} m$ magnetic symmetry with $m_{x}=m_{y}\left(F_{x y}\right)$. The inset shows indexed magnetic peaks, $\sqrt{2} a_{0} \times \sqrt{2} a_{0} \times 2 a_{0}$ setting. A schematic view of the magnetic structure is also shown.

the tetragonal setting) perpendicular to the vertical tetragonal axis. If $\left|m_{x}\right| \neq\left|m_{y}\right|$, the diagonal FM[110] order is split into two noncollinear sublattices that deviate from the diagonal line. Calling $\delta$ to the angle formed by the Co moments at $\left(0,0,0 \mid m_{x}, m_{y}, 0\right)$ and $\left(1 / 2,1 / 2,0 \mid m_{y}, m_{x}, 0\right)$ tetragonal sites, it is important to emphasize that the intensity of $(101) /(110)$ reflections would be proportional to $\delta$ if the moments were not collinear. Collinearity is here experimentally confirmed because these reflections have null intensity at $2 \theta=24.53^{\circ}$ in Fig. 5, leading to the diagonal $m_{x}=m_{y} \operatorname{model}\left(F_{x y}\right)$.

\section{Temperature evolution of the ordered FM moment (zero field NPD)}

The amplitude of the ordered FM moment per cobalt atom was refined as a function of temperature using the set of neutron patterns collected below $T_{c}$. Its evolution is shown in Fig. 6(a), using the $I m^{\prime} m^{\prime} a(F[100])$ and $F m^{\prime} m^{\prime} m$ $(F[110])$ magnetic models for, respectively, the orthorhombic FM2 and tetragonal FM1 phases. In this evolution, there is a small partial disruption of the ordered moment around $T_{S 1}$ (between $100 \mathrm{~K} \leqslant T \leqslant 130 \mathrm{~K}$ ), attributed to the natural disorder across the O-T structural transition. We do not observe the characteristic step down of the magnetization under very low fields. These results confirm the different evolution of the ordered atomic magnetic moment respect to the magnetization behavior.

The samples here presented were previously investigated by XMCD measurements at the Co $L_{2,3}$ edges [22], which revealed an unquenched orbital angular momentum in Co atoms. In Fig. 6(b), we reproduce (as adapted from XMCD data in Ref. [22]) the temperature dependence of the orbital magnetic moment $m_{L}$ under $0.1 \mathrm{~T}$. The $m_{L}(T)$ evolution showing an upturn in the figure contrasts with the typical behavior of the ordered FM moment deduced from neutrons and depicted in Fig. 6(a).
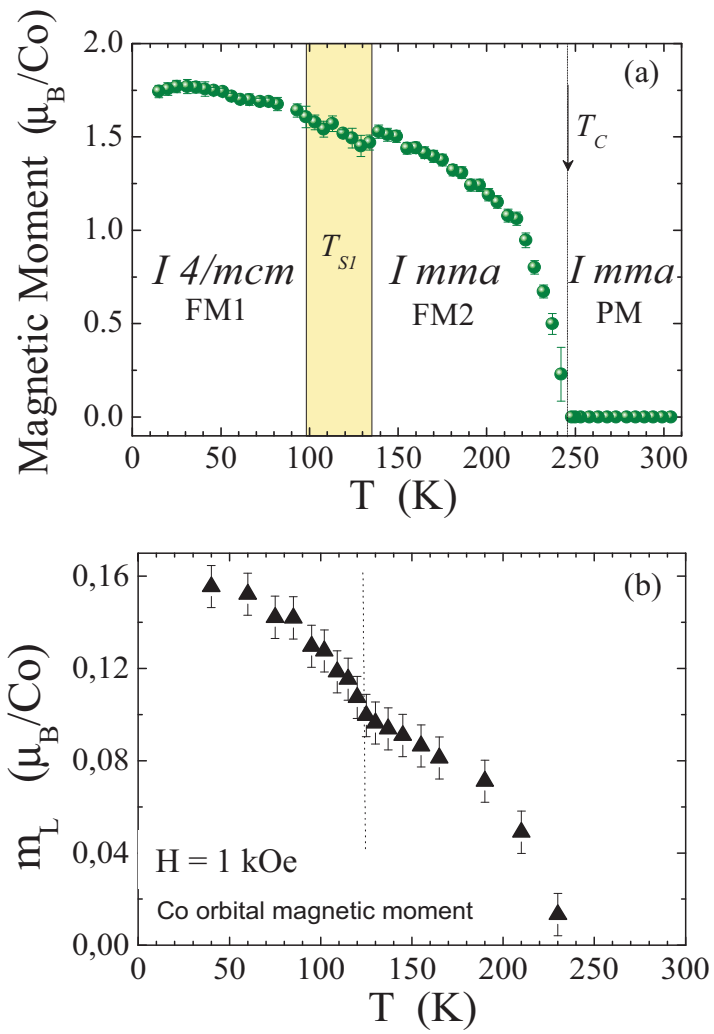

FIG. 6. (a) Temperature dependence of the ordered ferromagnetic moment per Co atom across the Curie and Imma to $I 4 / \mathrm{mcm}$ transitions obtained from the Rietveld refinement of neutron patterns. (b) Temperature dependence of the orbital magnetic moment $m_{L}$ (solid blue triangles) derived from XMCD spectra of PSCO at $0.1 \mathrm{~T}$ (adapted from Ref. [22]).

\section{NPD under magnetic field at $\mathbf{2 K}$}

Neutron diffraction experiments were extended to measurements under magnetic field in the low temperature FM1 phase. For that a cryomagnet was placed on the D1B powder diffractometer. A sintered cylindrical bar of PSCO was ZFC down to $2 \mathrm{~K}$. Then isothermal NPD measurements under field were carried out while increasing the vertically applied magnetic field up to a maximum value of $5 \mathrm{~T}$. The NPD patterns were collected under constant fields within the $0 \rightarrow 1 \mathrm{~T}$ $\left(\mu_{0} \Delta H=0.1 \mathrm{~T}\right.$ step $)$ and $1 \rightarrow 5 \mathrm{~T}(0.5 \mathrm{~T}$ step) intervals.

Figure 7 (left panel) displays the evolution upon increasing the magnetic field of the $(002) /(110)$ magnetic peaks at $2 \mathrm{~K}$. By simple inspection, one can see that the intensity of both reflections is very similar for $H=0$, whereas the applied field generates a visible increase of the (110) magnetic intensity with respect to the (002). Even so, the changes in the spectra are rather small. We confirmed that the application of moderate magnetic fields does not destabilize the $14 / \mathrm{mcm}$ structure. Following these observations, the neutron pattern obtained at $5 \mathrm{~T}$, was satisfactorily refined using the tetragonal crystal structure and FM Co moments with a component within the $a b$ plane $\left(F_{\perp z}\right)$ and an additional out-of-plane component $\left(F_{z}\right)$. The right panel of Fig. 7 plots the $(002) /(110)$ region of the Rietveld refinement of neutron data under $5 \mathrm{~T}$ at $2 \mathrm{~K}$ (a) constraining the FM moments to the $x y$ plane and (b) permitting also an out-of-plane component in the 

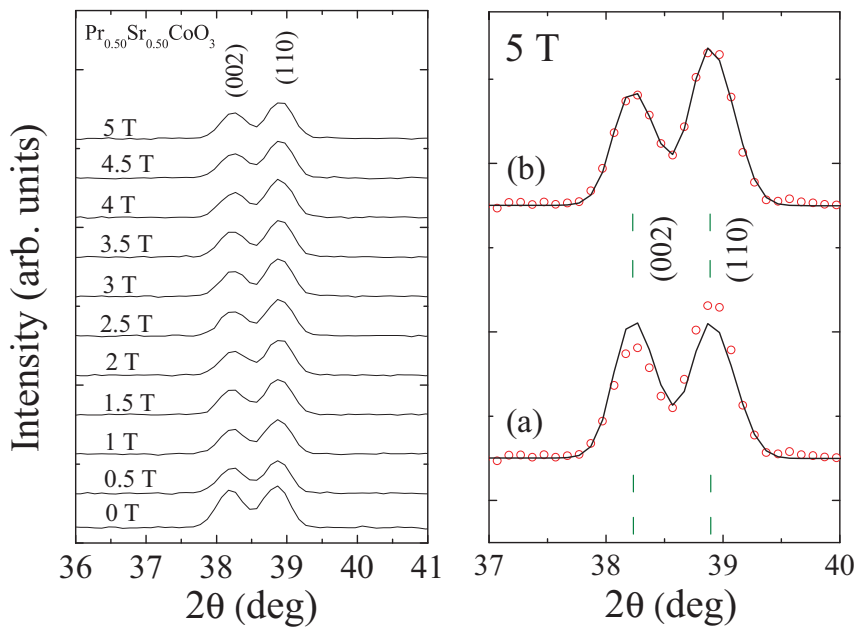

FIG. 7. PSCO, neutron powder diffraction at $2 \mathrm{~K}(\lambda=2.52 \AA)$. [Left panel] Evolution of the (002) and (110) reflections increasing the applied magnetic field (see explanation in the text). [Right panel] Selected angular region of the Rietveld refinements (solid line) at $2 \mathrm{~K}$ under $5 \mathrm{~T}$ corresponding to (a) $F_{\perp z}$ model (in-plane ferromagnetism) and (b) In-plane $\left(F_{\perp z}\right)+$ out-of-plane $\left(F_{z}\right)$ model with inclined FM moments deviated $28(2)^{\circ}$ from the plane. Patterns have been shifted up for clarity and are indexed in the tetragonal cell.

magnetization $\left(F_{x y} F_{z}\right)$. Only this last model correctly accounts for the experimental profile, and the best magnetic refinement under $5 \mathrm{~T}$ yields $m_{\mathrm{Co}}[5 \mathrm{~T}]=2.00(2) \mu_{\mathrm{B}} / \mathrm{Co}$ and $\theta=28(2)^{\circ}$ $\left(R_{B}=2.71, R_{f}=1.64, R_{\mathrm{Mag}}=1.95, \chi^{2}=1.36\right)$, where the angle $\theta$ defines the average deviation of the FM moments in the polycrystalline bar out of the $a b$ plane. Using the same model we have satisfactorily refined the successive neutron patterns recorded at fields between 0 and $5 \mathrm{~T}(2 \mathrm{~K})$. The two refined magnetic parameters (total atomic magnetic moment and the deviation angle $\theta$ out of the $a b$ plane) are depicted in Fig. 8 as a function of the external magnetic field. One observes that, upon increasing the applied field from zero, the inclination of the moment (angle $\theta$ ) first increases, but then it saturates reaching a constant value between 1.0 and $1.5 \mathrm{~T}$. So, the evolution of our polycrystalline sample shown in this figure indicates that an external field $\mu_{0} H_{z} \sim 1.2 \mathrm{~T}$ applied to a

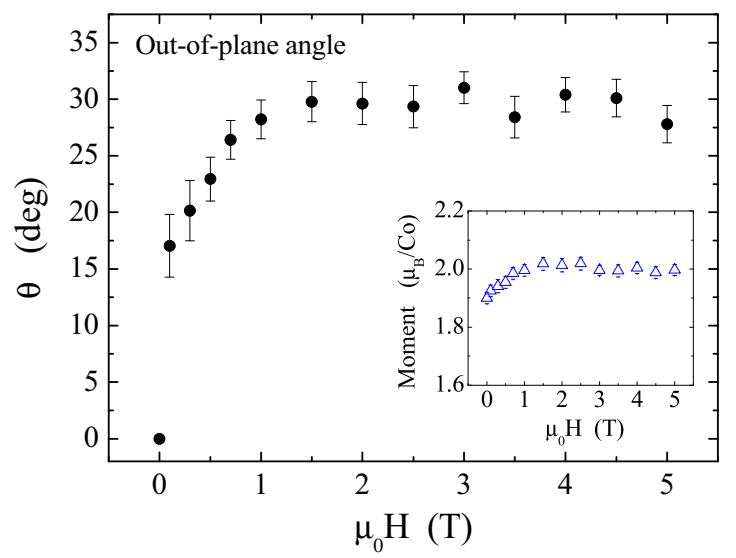

FIG. 8. Evolution upon increasing the magnetic field of the out-of-plane FM component in a polycrystalline bar of PSCO $(\theta$ : inclination angle). Inset: ordered FM moment per $\mathrm{Co}$ ion. single crystal in the FM2 phase would be enough to align all Co moments perpendicular to the plane (with $F_{z}$ configuration).

\section{CONCLUDING REMARKS}

In the previous sections, we have investigated the puzzling magnetic properties of the half-doped PSCO cobaltite. Even though the structural evolution with temperature was previously and extensively described in Ref. [18], its magnetic properties and the nature of the magnetostructural transition were not well understood. An orbital contribution to the magnetization (around 1/3 of the spin component) was revealed by XMCD, and the spin-orbit coupling term in this cobaltite promotes a parallel alignment of spin and orbital magnetic moments at both sides of the magnetostructural transition [22]. Very likely a reorientation of the orbital moment at $T_{S 1}$ is triggered by the Imma $\rightarrow I 4 / \mathrm{mcm}$ phase transition, and, through the spin-orbit coupling, the ordered Co spins rotate between the FM1 and FM2 phases. Indeed, two early reports suggested a rotation by $45^{\circ}$ of the magnetic easy axis: Hirahara et al. [20] reported preliminary magnetization measurements on a PSCO single crystal (described as monoclinic and only partially characterized); equally, LTEM images obtained by Uchida et al. [21] are concurrent with a $45^{\circ}$ rotation of the magnetization between $140 \mathrm{~K}$ and $80 \mathrm{~K}$ and with our neutron data analysis.

Together with previous LTEM results, the analysis of the magnetic structures using neutron diffraction confirms a spin reorientation accompanying the higher symmetry of the tetragonal $I 4 / \mathrm{mcm}$ cell. We have demonstrated that above $T_{S 1}$, the FM2 phase of PSCO is composed of [100] FM domains $\left(F_{x}\right)$, with magnetic symmetry $\operatorname{Im}^{\prime} m^{\prime} a\left(m_{x} \neq 0, m_{z}=0\right)$. For clarity, the coordinates $x, y, z$ are always referred to the setting of the tetragonal cell $\left(\sqrt{2} a_{0} \times \sqrt{2} a_{0} \times 2 a_{0}\right)$. Below $T_{S 1}$ there is a change in the magnetocrystalline axis. The coupled orbital and spin components of the moment rotate by $45^{\circ}$, and the easy axis aligns parallel to the diagonal of the tetragonal unit cell $\left(F_{x y}\right)$. The MSG of the low temperature phase is $F m^{\prime} m^{\prime} m$ (with $m_{x}=m_{y}$ ). The appearance of the fourfold tetragonal axis brings on degeneration and two equivalent magnetic easy axes, which are associated to the two conjugated magnetic domains with spin orientations [110] and [1-10]. Therefore, the loss of magnetization (negative step) under low fields $\left(H<H_{\mathrm{cr}}\right)$ is produced by the presence of conjugated [110] and [1-10] FM domains after the Imma $\rightarrow I 4 / m c m$ transition. A schematic view of magnetic ordering and magnetic domains above and below $T_{S 1}$ is shown in Fig. 9. For clarity reasons, time-reversal type domains are not shown in this figure. The coexistence of different types of domains at low temperatures depends on the sample history and the external applied field. On the other hand, the origin of the sudden positive jump in the magnetization under moderate fields could be ascribed to a larger $J / K_{a}$ ratio, with $J$ and $K_{a}$ being the double-exchange term and the magnetocrystalline energy, respectively. In the tetragonal phase, the Co-O-Co angle parallel to $c$ becomes completely flat and could favor the FM double exchange [18].

Recapitulating, the evolution of neutron diffraction data through the two successive magnetic transitions in $\mathrm{Pr}_{0.50} \mathrm{Sr}_{0.50} \mathrm{CoO}_{3}$ agrees with a reorientation of the easy axis of cobalt atoms favored by the Imma $\rightarrow I 4 / \mathrm{mcm}$ symmetry 
(a)

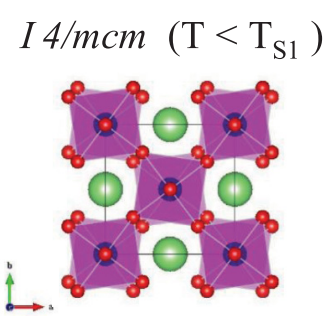

FM1

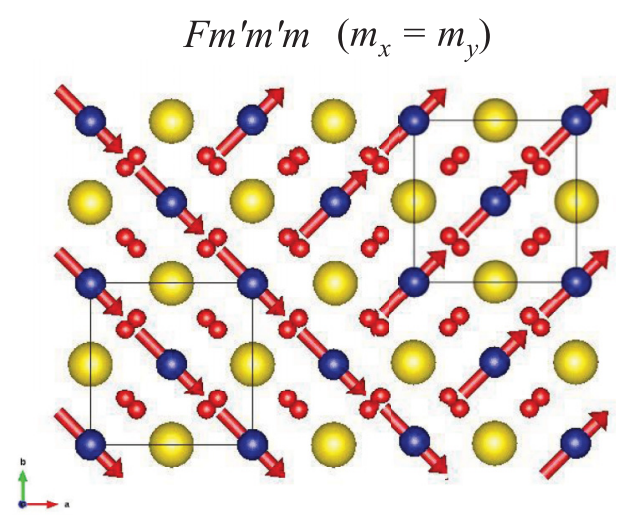

(b)

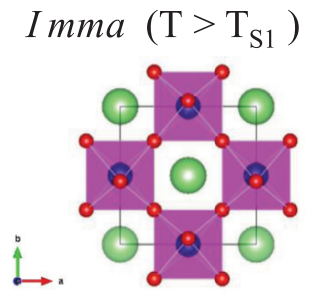

FM2

Im' $m^{\prime} a\left(m_{z}=0\right)$

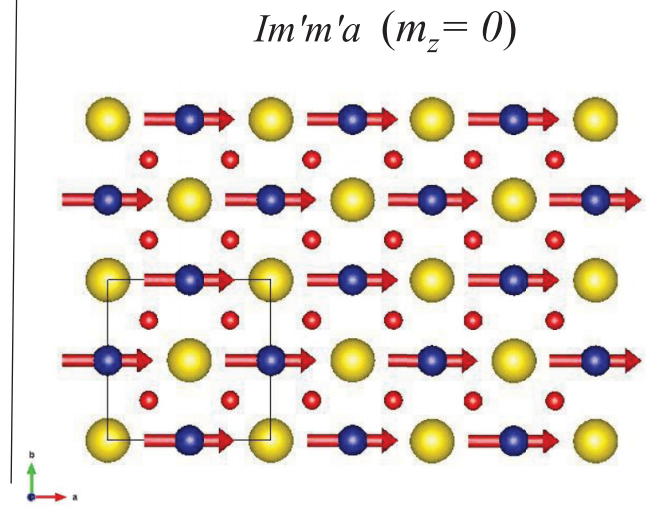

FIG. 9. Schematic view of the crystal and magnetic structures for (right) the ferromagnetic phase above $T_{S 1}$ (FM2) composed of [100] ferromagnetic domains and (left) the ferromagnetic phase below $T_{S 1}$ (FM1) showing the coexistence of conjugated [110] and [1-10] magnetic domains.

change at $T_{S 1}=120 \mathrm{~K}$. The magnetic symmetry $I m^{\prime} m^{\prime} a$ $\left(m_{x} \neq 0, m_{z}=0\right)$ in the orthorhombic phase below $T_{C}$ generates [100] type FM domains $\left(F_{x}\right)$. Neutron data analyses in combination with earlier reports agrees with a reorientation of the magnetization axis by $45^{\circ}$ within the $a b$ plane in the low temperature phase $\left(F_{x y}\right)$. The presence below $T_{S 1}$ of conjugated magnetic domains of $F m^{\prime} m^{\prime} m$ symmetry with spin orientations [110] and [1-10] $\left(\left|m_{x}\right|=\left|m_{y}\right|\right)$ is at the origin of the anomalies observed in the macroscopic magnetization. A relatively small field of $\mu_{0} H_{\mathrm{cr}}[\perp z] \approx 30 \mathrm{mT}$ is able to reorient the magnetization within the $a b$ plane, whereas a higher field $\left(\mu_{0} H_{\mathrm{cr}},[\| z] \sim 1.2 \mathrm{~T}\right.$ at $\left.2 \mathrm{~K}\right)$ would be necessary to align the Co moments perpendicular to this plane.

\section{ACKNOWLEDGMENTS}

We thank the spanish Ministerio de Economía y Competitividad (MINECO) for financial support under Projects No. MAT2012-38213-C02-02 and -01 and No. MAT201568760-C2-2-P and -1-P (cofunded by the European Regional Development Fund [ERDF] from the European Union) and No. CSD2007-00041 (NANOSELECT). We also acknowledge ILL and ALBA for granting beamtime. C. Ritter is acknowledged for technical assistance during neutron measurements. J.L.G.-M. thanks J. M. Pérez-Mato for fruitful discussions. J.P.-P. and J.A.R.V. thank CSIC for JAE-Pre and JAE-Doc contracts, respectively.
[1] S. Tsubouchi, T. Kyômen, M. Itoh, P. Ganguly, M. Oguni, Y. Shimojo, Y. Morii, and Y. Ishii, Phys. Rev. B 66, 052418 (2002).

[2] C. Frontera, J. L. García-Muñoz, A. Llobet, and M. A. G.Aranda, Phys. Rev. B 65, 180405(R) (2002); C. Frontera, J. L. GarcíaMuñoz, A. E. Carrillo, M. A. G. Aranda, I. Margiolaki, and A. Caneiro, J. Solid State Chem. 171, 349 (2003).

[3] A. Maignan, V. Caignaert, B. Raveau, D. Khomskii, and G. Sawatzky, Phys. Rev. Lett. 93, 026401 (2004); C. Frontera, J. L. García-Muñoz, A. E. Carrillo, M. A. G. Aranda, I. Margiolaki, and A. Caneiro, Phys. Rev. B 74, 054406 (2006).

[4] A. J. Barón-González, J. L. García-Muñoz, J. Herrero-Martín, C. Frontera, G. Subías, and J. Blasco, J. Kor. Phys. Soc. 63, 791 (2013).

[5] A. J. Barón-González, C. Frontera, J. L. García-Muñoz, J. Blasco, and C. Ritter, Phys. Rev. B 81, 054427 (2010).
[6] J. Hejtmánek, E. Šantavá, K. Knížek, M. Maryško, Z. Jirák, T. Naito, H. Sasaki, and H. Fujishiro, Phys. Rev. B 82, 165107 (2010).

[7] K. Knížek, J. Hejtmánek, P. Novák, and Z. Jirák, Phys. Rev. B 81, 155113 (2010).

[8] J. L. García-Muñoz, C. Frontera, A. J. Barón-González, S. Valencia, J. Blasco, R. Feyerherm, E. Dudzik, R. Abrudan, and F. Radu, Phys. Rev. B 84, 045104 (2011).

[9] J. Herrero-Martín, J. L. García-Muñoz, S. Valencia, C. Frontera, J. Blasco, A. J. Barón-González, G. Subías, R. Abrudan, F. Radu, E. Dudzik, and R. Feyerherm, Phys. Rev. B 84, 115131 (2011).

[10] J. Herrero-Martín, J. L. García-Muñoz, K. Kvashnina, E. Gallo, G. Subías, J. A. Alonso, and A. J. Barón-González, Phys. Rev. B 86, 125106 (2012). 
[11] Y. Okimoto, X. Peng, M. Tamura, T. Morita, K. Onda, T. Ishikawa, S. Koshihara, N. Todoroki, T. Kyomen, and M. Itoh, Phys. Rev. Lett. 103, 027402 (2009).

[12] R. Mahendiran and P. Schiffer, Phys. Rev. B 68, 024427 (2003).

[13] C. Leighton, D. D. Stauffer, Q. Huang, Y. Ren, S. El-Khatib, M. A. Torija, J. Wu, J. W. Lynn, L. Wang, N. A. Frey, H. Srikanth, J. E. Davies, Kai Liu, and J. F. Mitchell, Phys. Rev. B 79, 214420 (2009).

[14] N. A. Frey Huls, N. S. Bingham, M. H. Phan, H. Srikanth, D. D. Stauffer, and C. Leighton, Phys. Rev. B 83, 024406 (2011).

[15] I. O. Troyanchuk, D. V. Karpinskii, A. N. Chobot, D. G. Voitsekhovich, and V. M. Bobryanskii, JETP Lett. 84, 151 (2006).

[16] A. M. Balagurov, I. A. Bobrikov, V. Y. Pomjakushin, E. V. Pomjakushina, D. V. Sheptyakov, and I. O. Troyanchuk, JETP Lett. 93, 263 (2011); A. M. Balagurov, I. A. Bobrikov, D. V. Karpinsky, I. O. Troyanchuk, V. Y. Pomjakushin, and D. V. Sheptyakov, ibid. 88, 531 (2008).

[17] F. Li, N. Wu, and J. Fang, J. Supercond. Nov. Magn. 26, 463 (2013).

[18] J. Padilla-Pantoja, J. L. García-Muñoz, B. Bozzo, Z. Jirák, and J. Herrero-Martín, Inorg. Chem. 53, 12297 (2014).
[19] J. Padilla-Pantoja, J. Herrero-Martín, P. Gargiani, M. Valvidares, V. Cuartero, K. Kummer, O. Watson, N. Brookes, and J. L. García-Muñoz, Inorg. Chem. 53, 8854 (2014).

[20] S. Hirahara, Y. Nakai, K. Miyoshi, K. Fujiwara, and J. Takeuchi, J. Magn. Magn. Mater. 310, 1866 (2006).

[21] S. Uchida, R. Mahendiran, Y. Tomioka, Y. Matsui, K. Ishizuka, and Y. Tokura, Appl. Phys. Lett. 86, 131913 (2005).

[22] J. Padilla-Pantoja, J. Herrero-Martín, E. Pellegrin, P. Gargiani, S. M. Valvidares, A. Barla, and J. L. García-Muñoz, Phys. Rev. B 92, 245136 (2015).

[23] J. Rodríguez-Carvajal, Physica B 192, 55 (1993); http://www.ill.eu/sites/fullprof/.

[24] M. I. Aroyo, J. M. Perez-Mato, C. Capillas, E. Kroumova, S. Ivantchev, G. Madariaga, A. Kirov, and H. Wondratschek, Z. Kristallogr. 221, 15 (2006); http://www.cryst.ehu.es.

[25] M. I. Aroyo, A. Kirov, C. Capillas, J. M. Perez-Mato, and H. Wondratschek, Acta Crystallogr. Sect. A 62, 115 (2006).

[26] J. M. Perez-Mato, S. V. Gallego, E. S. Tasci, L. Elcoro, G. de la Flor, and M. I. Aroyo, Annu. Rev. Mater. Res. 45, 217 (2015).

[27] N. V. Belov, N. N. Neronova, and T. S. Smirnova, Kristallografiya 2, 315 (1957) [Sov. Phys. Crystallogr. 2, 311 (1957)].

[28] F. Damay, C. Martin, M. Hervieu, A. Maignan, B. Raveau, G. Andre, and F. Boure, J. Magn. Magn. Mater. 184, 71 (1998). 